\title{
Molecular cloning and mRNA expression of a hepcidin gene from the spinyhead croaker, Collichthys lucidus
}

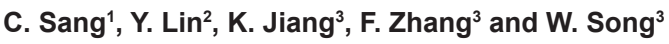 \\ ${ }^{1}$ School of Ocean, Yantai University, Yantai, Shangdong, China \\ ${ }^{2}$ College of Fisheries and Life Science, Shanghai Ocean University, \\ Shanghai, China \\ ${ }^{3}$ East China Sea Fisheries Research Institute, Chinese Academy of Fishery Sciences, \\ Shanghai, China
}

Corresponding author: W. Song

E-mail: songw@ecsf.ac.cn

Genet. Mol. Res. 14 (4): 16050-16059 (2015)

Received August 16, 2015

Accepted October 14, 2015

Published December 7, 2015

DOI http://dx.doi.org/10.4238/2015.December.7.18

\begin{abstract}
Antimicrobial peptides are important components that participate in host innate immune activities and play crucial roles in host defense against microbial invasion. Hepcidin is an antimicrobial peptide and iron-regulatory molecule that primarily functions in the liver. In the present study, we first obtained a full-length cDNA sequence of hepcidin and its corresponding genomic DNA sequence from Collichthys lucidus using RTPCR and rapid amplification of CDNA ends (RACE), and then analyzed these sequences using bioinformatics software. The results showed that $C$. lucidus hepcidin (CL-hepc) possesses two introns and three exons in the genomic DNA, with a length of $816 \mathrm{bp}$. The open reading frame was 264 bp, encoding an 87 amino acid peptide, and with high similarity (88.89\%) to 83416593 Larimichthys crocea (ABC18307) and relatively low similarity $(47.73 \%$ ) to 158358729 L. crocea (ABY84845.1). The pre-peptide contained a signal peptide (28 amino acids), a prodomain (34 amino acids), and a mature peptide ( 25 amino acids). The predicted 25 amino acid hepcidin
\end{abstract}


mature peptide included 8 conserved cysteine residues. Quantitative real-time reverse transcription-PCR analysis revealed specific expression patterns of CL-hepc, with the highest expression observed in the liver, relatively low expression observed in the gill and spleen, and almost no expression detected in other tissues analyzed. In conclusion, we identified a hepcidin from $C$. lucidus that has common expression patterns with other hepcidins. However, as this hepcidin is inconsistent with two other hepcidins from L. crocea in terms of the phylogenetic tree, the presence of another hepcidin gene warrants further investigation.

Key words: Hepcidin; Collichthys Iucidus; Gene clone; Innate immune

\section{INTRODUCTION}

Hepcidin (HEPC), a cysteine-rich antimicrobial peptide, was first discovered in human plasma ultrafiltrate (Krause et al., 2000) and urine (Park et al., 2001), and subsequently the sequences were identified from various vertebrates, including mice (Pigeon et al., 2001), dog (Fry et al., 2004), swine (Sang et al., 2006), pigeons (Fu et al., 2007), equine (Oliveira Filho et al., 2010), and zebrafish (Shike et al., 2004). In vertebrates, hepcidin is mainly involved in the innate immune system and maintenance of iron homeostasis (Park et al., 2001; Nemeth et al., 2003). Its synthesis is greatly stimulated by inflammation or iron overload. In anemia or inflammation, HEPC production is increased by approximately 100 -fold, which may account for the defining feature of this condition, sequestration of iron in macrophages (Ganz, 2003). Ferroportin is an iron exporter present on the surface of absorptive enterocytes, macrophages, hepatocytes, and placental cells. HEPC binds to ferroportin to induce its degradation, thus, reducing the exportation of iron. Through this pathway, homeostasis of the iron system is regulated, i.e., iron regulates the secretion of HEPC, which in turn controls the concentration of ferroportin on the cell surface (Nemeth et al., 2004). The key role of HEPC suggests that drugs acting as HEPC agonists or antagonists may be a potential target for the treatment of iron-related diseases (Nicolas et al., 2002). In addition, studies on human HEPC have demonstrated that HEPC has a relatively wide antibacterial spectrum, including fungi, such as Candida albicans, Aspergillus fumigatus, and Aspergillus niger, and bacteria, such as Escherichia coli, Staphylococcus aureus, Staphylococcus epidermidis, and group B Streptococcus.

Due to the lack of a well-developed adaptive immune system, fish rely heavily on innate components when encountering various pathogens (Magnadottir, 2006). The sequences of HEPCs have been cloned in many fish species, including both freshwater fish, such as zebrafish (Shike et al., 2004), mud loach (Nam et al., 2011), and rice field eel (Li et al., 2011), and marine fish, including Epinephelus coioides (Qu et al., 2013), Pseudosciaena crocea (Wang et al., 2009), Cynoglossus semilaevis (Wang et al., 2012), rainbow trout (Alvarez et al., 2013), and Chrysophrys major (Chen et al., 2005). HEPC in fish has a similar structure and mRNA distribution to other species, such as mammals, although there are some differences. In zebra fish (Shike et al., 2004) and channel catfish (Bao et al., 2005), the transcripts of HEPC are mostly found in other tissues rather than in the liver. In addition, no alteration in expression level was detected in iron-deficient fish when they were submitted to either iron status modulation or bacterial infection (Rodrigues et al., 2006). HEPC peptides, including purified natural peptides (Shike et al., 2002), recombinant fusion HEPC (Bao et al., 2005), and synthesized peptides (Wang et al., 2009) all exhibited an effective activity against 
different species of microorganisms. Meanwhile, the antimicrobial spectrum of HEPC activity from different fish species appeared to be individually specific, suggesting that different HEPCs from different sources may have specific antimicrobial action (Wang et al., 2009). In addition, the use of HEPC antibacterial peptide from large yellow croaker as a feed additive could prevent disease and treat sea fish (Wang et al., 2011), suggesting that obtaining various HEPCs from different species may sometimes be promising for the treatment of various diseases.

Collichthys lucidus Richardson (Perciformes, Sciaenidae, Collichthys), is a commercially important near-shore species widely distributed in the South Sea and the East Sea of China in particular (Cheng et al., 2012). Based on its excellent properties and quality of meat, C. lucidus is highly valued by Chinese consumers. Knowing that resources of $C$. lucidus are declining as a result of increasingly serious problems, such as pollution and over-fishing, several institutions in China, including the East China Sea Fisheries Institute and Shanghai Fisheries Research Institute, have begun to study the artificial propagation of $C$. lucidus, with substantial progress being made recently. The aim of the present study was to characterize both the mRNA and genome DNA sequences of $C$. Iucidus HEPC (CL-hepc) and analyze expression patterns in different tissues in an attempt to lay a preliminary foundation for promoting aquaculture of $C$. lucidus and other species.

\section{MATERIAL AND METHODS}

\section{Determination of CL-hepc cDNA and genomic DNA sequences}

\section{Construction of the cDNA library and cloning of CL-hepc cDNA from C. Iucidus}

Healthy fish weighing $35 \pm 5 \mathrm{~g}$ were captured from the East China Sea area near Shanghai, China. Tissue samples $(200 \pm 50 \mathrm{mg})$ for RNA extraction were obtained through vivisection on fishing boats and then stored in a $1.5 \mathrm{~mL}$ RNA fixer (Bioteke Corporation, Beijing, China) at $-20^{\circ} \mathrm{C}$. Total RNA was isolated from the hepatopancreas, gill, heart, muscle, and blood of $C$. lucidus using TRIpure reagent (Aidlab, Beijing, China) following the manufacturer protocol. Quality and concentration of RNA were checked using agarose gel electrophoresis (Universal Hood II, Bio-Rad Laboratories Inc., Hercules, CA, USA) and spectrophotometry (DU800 Nucleic acid/Protein Analyzer, Beckman Coulter, Brea, CA, USA). RNA was stored at $-80^{\circ} \mathrm{C}$ until use.

A cDNA library was constructed successfully using a SMART ${ }^{\mathrm{TM}}$ CDNA library construction kit (TaKaRa, Dalian, China), followed by transformation, screening, and sequencing. Random sequencing was performed after cDNA library construction. All expressed sequence tags were subjected to BLAST analysis.

A cDNA sequence comprising the CL-hepc domain was obtained and subjected to further investigation. RACE (3'-rapid amplification of cDNA ends) was carried out following the manufacturer protocol using the 3'-full RACE core set (TaKaRa) to obtain the full-length cDNA sequence. Two gene specific-primers, CL-hepc-outer (5'-ACTCGTGCTCGCCTTTATTTGC-3') as the 3'-RACE amplification outer primer and CL-hepc-inner (5'-TTCTGAGGATTCCCAGCGACGACCAA-3') as the nest PCR primer, were designed according to the obtained sequence and used for 3'-RACE. PCR was carried out following the manufacturer protocol. The PCR products were checked on a $1.0 \%$ agarose gel and purified using the Agarose Gel DNA Purification kit v2.0. (TaKaRa), and then cloned into the pMD19-T vector (TaKaRa) for sequencing. 


\section{Genomic DNA determination of CL-hepc}

Genomic DNA was isolated from the muscle of $C$. Iucidus using the TIANamp Marine Animals DNA kit (TIANGEN, Beijing, China) according to the manufacturer protocol. Primers CL-hepc-g-F (5'-GAAGAAATCCTCTTGCCTGGACG-3') and CL-hepc-g-R (5'-AAGGTCATTTCCTGGGGTGAG ATAC-3') were designed based on the 5'-untranslated region (UTR) and the $3^{\prime}-\mathrm{UTR}$ of the cDNA sequences.

\section{Bioinformatic analysis}

General features of the CL-hepc full-length cDNA were detected by Vector NTI Advance 11.5 (http://www.lifetechnologies.com/cn/zh/home/life-science/cloning/vector-nti-software/vectornti-advance-software/whats-new-in-vector-nti-advance.html), and the peptide sequence was deduced and verified according to other HEPCs using the NCBI Open Reading Frame (ORF) Finder (http://www.ncbi.nlm.nih.gov/gorf/gorf.html). The biochemical features of the deduced protein were calculated using the Expert Protein Analysis System (http://web.expasy.org/). Amino acid sequences from various species were obtained from NCBI GenBank and analyzed using the MEGA software version 5.0 (Saitou and Nei, 1987). Phylogenetic analysis was performed using the neighbor-joining method using the MEGA software version 5.0 and the confidence level in the generated phylogenetic tree was obtained using 1000 bootstraps. The secondary structure of the CL-hepc protein was predicted using the Predict Protein package (available online at http://www. predictprotein.org/).

\section{Tissue distribution analysis of CL-hepc mRNA in adults}

Tissue samples, including the liver, blood, heart, muscle, gill, spleen, brain, and kidney, were collected from fish using the same method as described above, from which total RNA (approximately $1 \mu \mathrm{g}$ ) was extracted and reverse transcribed using the quantitative real-time PCR (qRT-PCR) kit (TOYOBO, Japan) for first-strand cDNA synthesis. The products were diluted 10 times and stored at $-20^{\circ} \mathrm{C}$ until qRT-PCR analysis.

The qRT-PCR assay was carried out in a StepOne Plus detection system (Applied Biosystems). The transcript expression profiles of CL-hepc in eight different tissues of $C$. lucidus were detected using a pair of CL-hepc specific primers (CL-hepc-RT-F: 5'-GAAACGTGGATGCCACAT TTCA-3' and CL-hepc-RT-R: 5'-GAAGCCGCAGCCTTTGTTCTT-3'), and an 18S rRNA primer pair (18S-RT-F: 5'-GCCTGAATACCGCAGCTAGGAATAA-3' and 18S-RT-R: 5'-TTTCACCTCTAGCGG CACAATACG-3') designed from the 18S rRNA sequence on NCBI GenBank (accession No. JN211725.1) used as the internal control. CL-hepc expression levels were calculated using the standard curve method (Sun et al., 2012). Amplifications were performed on a 96-well plate with a $20-\mu \mathrm{L}$ reaction volume containing $10 \mu \mathrm{L} 2$ X Power SYBR Green PCR Master Mix (TaKaRa, Applied Biosystems), $1.0 \mu \mathrm{L}$ PCR forward primer $(10 \mu \mathrm{M}), 1.0 \mu \mathrm{L}$ PCR reverse primer $(10 \mu \mathrm{M})$, $2.0 \mu \mathrm{L}$ cDNA template, and $6 \mu \mathrm{L}$ diethylpyrocarbonate-treated water (DEPC-treated water). The reaction process for qRT-PCR was $10 \mathrm{~min}$ at $95^{\circ} \mathrm{C}$, followed by 40 cycles of $95^{\circ} \mathrm{C}$ for $15 \mathrm{~s}$ and $60^{\circ} \mathrm{C}$ for $1 \mathrm{~min}$. 


\section{RESULTS}

\section{Determination of CL-hepc cDNA and genomic DNA sequences}

The full-length cDNA of CL-hepc was 816 bp in length, containing a 264-bp ORF encoding a protein of 87 amino acids, and CL-hepc included a 5'-UTR of $201 \mathrm{bp}$ and a 3'-UTR of $351 \mathrm{bp}$, including a poly $(A)+$ tail. A typical poly-adenylation signal (AATAAA) was located 17 bp upstream of the poly $(A)+$ tail. The signal peptide cleavage site of the deduced CL-hepc was predicted between Ala 24 and Val 25 (Figure 1). According to the conserved motif "RQKR", the mature peptide of CL-hepc contained 25 amino acids that started with GIn 63. Thus, the mature peptide consisted of 25-amino acid residues at the $C$ terminus. The predicted molecular weight of CL-hepc was 2876.9 Da with an isoelectric point of 8.41. Meanwhile, the mature peptide had the typical eightCys structure, which is a common feature of the HEPC family.

\begin{tabular}{|c|c|}
\hline & (Exon 1) \\
\hline 1 & CAGCAGGAAGGAGTTGACAAGGGTCACCCAAAGATCTGAAGAAATCCTCTTGCCTGGACG \\
\hline 61 & ATCACCATCCATCACTGGAGCTGAAAAAATAAATTGAAGTTATTGTGGTGCTCTTTGGTG \\
\hline 121 & GCCCATGAGAAAAAAGACCCACCAGGTCTAATCTGCAAAGGATTTATTAACTAAACCATT \\
\hline 181 & TTTTGAAAAAGAAAAGCTAAAATGAAGGCATTCAGCATTGCAGTTGCAGTGACACTCGTG \\
\hline \multirow[t]{2}{*}{1} & 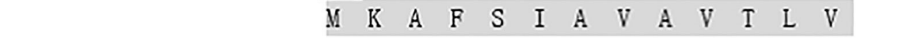 \\
\hline & (Intron 1) \\
\hline 241 & CTCGCCTTTATTTGCATTCIGGAGAGCTCTGCCGTCCCATTCACCGGG g taagaatccga \\
\hline \multirow[t]{2}{*}{14} & 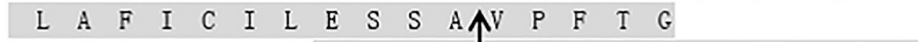 \\
\hline & Predicated cleavage site for signal peptide \\
\hline 301 & $\begin{array}{l}\text { cttaaactcacttcattgct tattagctataaatgtttgtcaggatgctgagatccta } \\
(\text { Exon } 2)\end{array}$ \\
\hline 361 & aatgtgcgtaat tcattaacagGTGCAGGAGCTGGAGGAGGCAGGGAGCAATGACACT \\
\hline \multirow[t]{2}{*}{30} & $\begin{array}{lllllllllllll} & V & Q & E & L & E & E & A & G & S & N & D & T\end{array}$ \\
\hline & (Intron 2) \\
\hline 419 & CCAGTTGCGGCGCATCAAGAATCGTCAAAGGAAACGTGGATGgtatgttcaattgactga \\
\hline 42 & 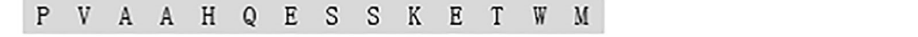 \\
\hline 479 & atgaat taagccaattactgtgagcaaattaaaatt taagtggatgtgttttccccacgg \\
\hline 539 & $\begin{array}{l}\text { agtatcctggtgctctctccgcacaaggagaggatgaaatgctgggtgatggaacgccac } \\
(\text { Exon } 3)\end{array}$ \\
\hline 599 & gctcatgtctct tgtctttttgcacagCCACATTTCAGACAGAAGCGOCAGAGCCACATC \\
\hline \multirow[t]{2}{*}{56} & $\begin{array}{lllllllllllllll} & P & H & F & R & Q & K & R & Q & S & H & I\end{array}$ \\
\hline & Predicted processing site for mature peptide \\
\hline 659 & TCCTTGTGCCGCTACTGCTGCAACTGCTGCAAGAACAAAGGCTGOGGCTTCTGCTGCAGG \\
\hline 67 & 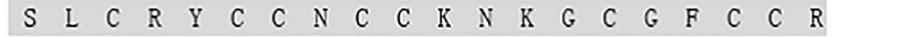 \\
\hline 719 & TTCTGAGGATTOCCAGCGACGACCAACAAATATTAATTTATTATGCTTTGTATCTCACCC \\
\hline 87 & * \\
\hline 779 & CAGGAAATGAOCTTTTCTTGACTTTTTTTTTTCTTTCTCCAATGCATTGCCTGTGCCACA \\
\hline 839 & CTGTTTTGTATCGTTTTCATGAGGTATGATTCATGGAAGAAGCACTGGAAAAGAAATGAA \\
\hline 899 & GCTATTCTTTGTATTTATTTTGCATGTTTTCATACTTCCGTCCCCGGAAGTCTTTTTTTG \\
\hline 959 & TAAACGCAACACTTTGTAAATT TGTTTCTAGAAATACAGATGTGACTGTTTCTTTATCTA \\
\hline 1019 & AATCAAATTCATTTTTGATGCTGAATAAAAAATGAACATTCAAAGCAAAAAAAAAAA \\
\hline
\end{tabular}

Figure 1. Nucleotide sequence of CDNA, predicted amino acid sequences, and genomic DNA organization of spinyhead croaker HEPC (CL-hepc). The exon sequence is in uppercase and the intron sequence is in lowercase. The deduced amino acid sequences are translated and shadowed. Vertical arrows show the predicted positions of cleavage sites for signal peptides and mature peptides and the box indicates the putative motif RQKR. The start codon (ATG) and stop codon (TGA) are bold and underlined, the polyadenylation signal (AATAAA) is bold. 
The CL-hepc genomic DNA sequence was amplified and sequenced and the C. lucidus HEPC gene was found to consist of two introns (94 and $165 \mathrm{bp}$ ) and three exons (Figure 1), which is identical to many other HEPCs in terms of genome structure. The first exon contained the 5'-UTR, the signal peptide, and 5-amino acid residues of the prodomain peptide. The prodomain extended from exon 1 through exon 3 . Two introns were inserted into the prodomain, thus, separating the peptide into three exons. Exon 3 encoded the mature peptide and the 3'-UTR. CL-hepc genomic organization is indicated in Figure 1.

Twenty-five amino acid sequences of various species were obtained using "HEPC" in a key word search of the protein database (http://www.ncbi.nlm.nih.gov/guide/proteins/\#databases). All sequences were aligned using ClustalW in the MEGA 5.0 software. All sequences contained eight Cys domains in the mature peptide located in the C-terminal (Figure 2). In addition, most sequences contained a conserved $\mathrm{RX}(\mathrm{K} / \mathrm{R}) \mathrm{R}$ domain that could be recognized by the precursor peptidase. Signal peptides are highly conversed in fish, as they are in mammals. Of the 25 sequences obtained through the database search, HEPC from 83416593 Larimichthys crocea had the highest similarity (88.89\%) with CL-hepc, followed by Micropterus dolomieu (87.78\%) and Pogonophryne scotti (83.33\%). In addition, HEPC from the Danio rerio isoform1 had $39.51 \%$ similarity with CL-hepc and $23.60 \%$ with Homo sapiens.

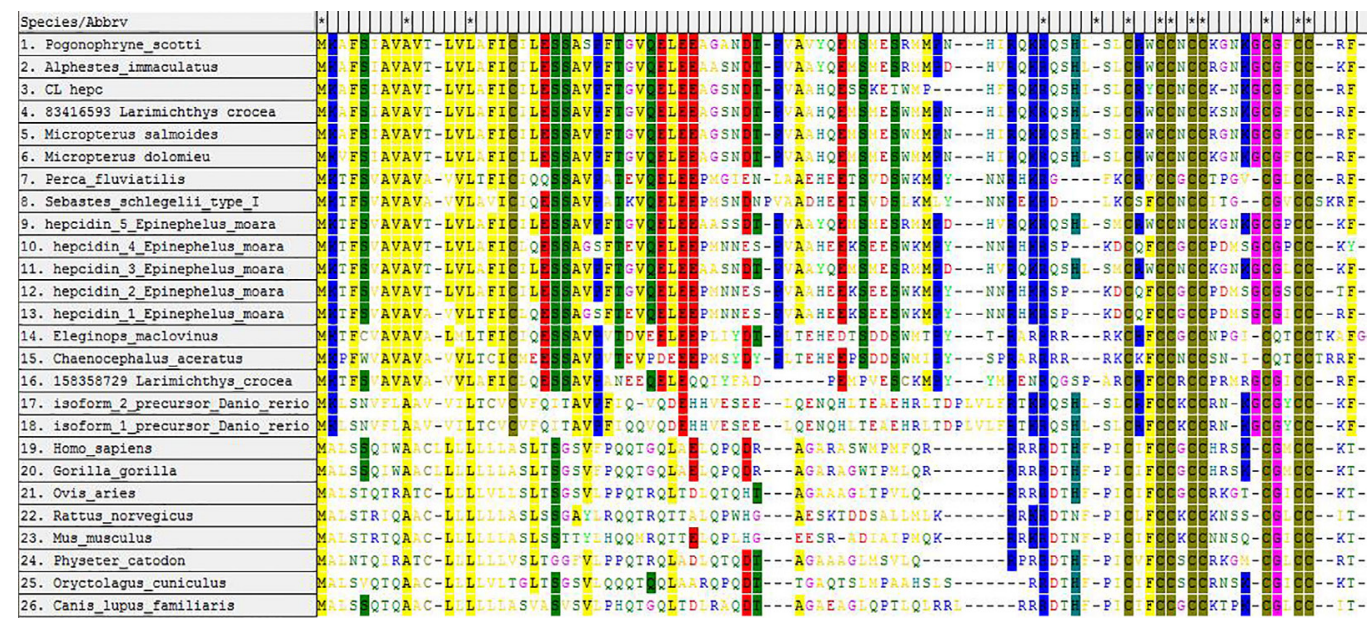

Figure 2. Clustal $\mathrm{W}$ alignment and comparison of the amino acid sequences deduced from Collichthys lucidus HEPC cDNA with some similar protein sequences of HEPC. Similarities of more than $50 \%$ are labeled in different colors. The amino acid sequence deduced from spinyhead croaker is marked as CL-hepc. Other similar proteins predicted and obtained from GenBank are as follows: Pogonophryne scotti (ABY84821.1); Alphestes immaculatus (AER00228.1); 158358729 Larimichthys crocea (ABY84845.1); 83416593 L. crocea (ABC18307); Micropterus salmoides (ACD13027.1); Micropterus dolomieu (ACD13029.1); Perca fluviatilis (ABR04075.1); Sebastes schlegelii type I (ACD80122.1); HEPC 5 Epinephelus moara (ADY16665.1); HEPC 4 E. moara (ADY16664.1); HEPC 3 E. moara (ADY16663.1); HEPC 1 E. moara (ADY16661.1); HEPC 2 E. moara (ADY16662.1); Eleginops maclovinus (ABY84826.1); Chaenocephalus aceratus (ABY84828.1); HEPC isoform 2 Danio rerio (NP_001276723.1); HEPC1soform 1 D. rerio (NP_991146.1); Homo sapiens (AAH20612.1); Gorilla gorilla (ABU75219.1); Ovis aries (ADK56130.1); Rattus norvegicus (NP_445921.1); Mus musculus (NP_115930.1); Physeter catodon (XP_007106594.1); Oryctolagus cuniculus (XP_0082477̄17.1); and Canis lupus familiaris (AAT95397.1). 
Phylogenetic analysis carried out using the bootstrap test of phylogeny produced an NJphylogenetic tree (Figure 3). The analysis involved all 26 aligned amino acid sequences. Based on the tree, HEPCs were separated into two clusters, one for fish and the other for mammals. The fish cluster was further divided into three branches. CL-hepc has the closest relationship with 83416593 L. crocea, thus, supporting the traditional taxonomic relationship. Interestingly, CL-hepc has a relatively distant relationship with another type of HEPC from L. crocea (ABY84845.1).

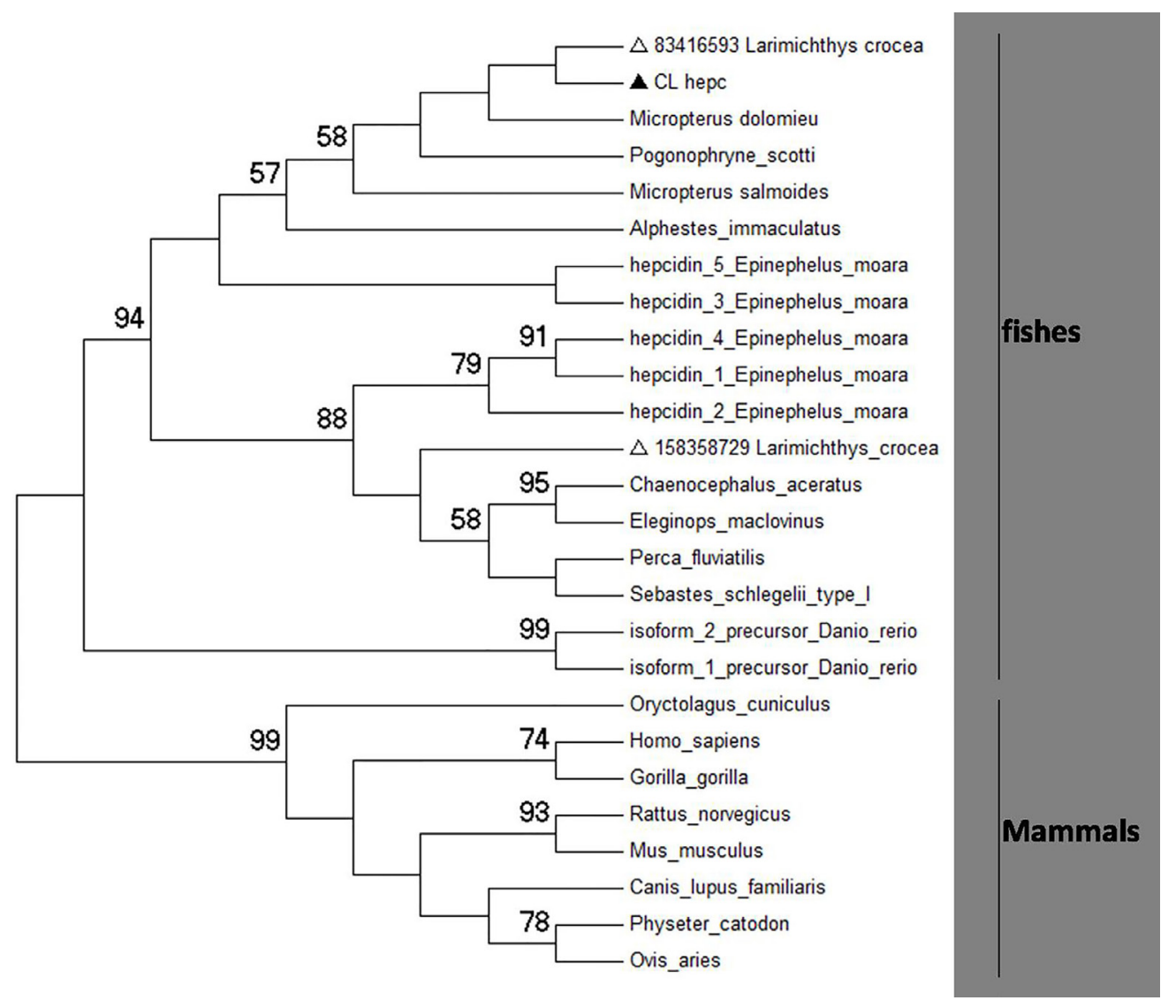

Figure 3. Phylogenetic tree based on HEPC amino acid sequences. The tree was constructed using the neighborjoining (NJ) algorithm in the MEGA 5.1 program based on multiple-sequence alignment by Clustal W. CL-hepc denotes Collichthys lucidus HEPC. Numbers at nodes indicate bootstrap values greater than $50 \%$ with 1000 replicates.

\section{Relative expression of CL-hepc in different tissues}

The relative expression of CL-hepc in the liver, blood, heart, muscle, gill, spleen, brain, and kidney was investigated using quantitative real-time PCR. Expression was found to be the highest in the liver and was significantly higher than that observed in other tissues, followed by the kidney and gill. There was no or little expression in the heart, spleen, blood, and brain (Figure 4). 


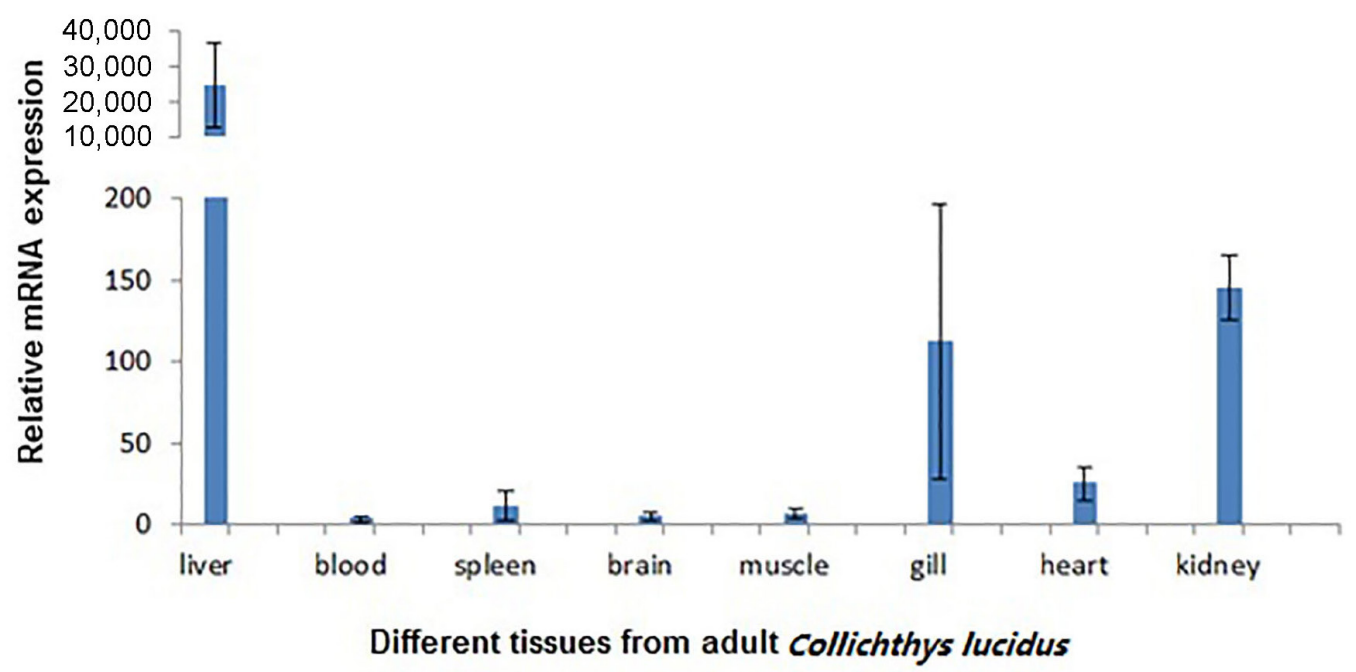

Figure 4. Expression profiles of the HEPC (CL-hepc) gene in different tissues of Collichthys lucidus detected by quantitative real-time PCR. The quantity of CL-hepc mRNA was normalized to the $18 \mathrm{~S}$ rRNA transcript level. The $y$-axis represents the relative ratio of expression levels of CL-hepc/18S rRNA.

\section{DISCUSSION}

The full-length sequence of CL-hepc is $816 \mathrm{bp}$, containing a 264-bp ORF encoding a protein of 87 amino acids. The cDNA has an 11-bp poly $(A)+$ tail with a typical poly-adenylation signal (AATAAA) located $17 \mathrm{bp}$ upstream. The predicted signal cleavage site of CL-hepc is between Ala24 and Val25, which is similar to that in Micropterus salmoides (Robertson et al., 2009) and $P$. crocea (Wang et al., 2009). The amino acid sequence of CL-hepc possesses the conserved motif "RQKR" that can be catalyzed by furin (Hosaka et al., 1991), thus, the mature peptide of CL-hepc contains 25 amino acids and starts with Gln 63, and has the typical eight-Cys structure, which is a common feature of the HEPC family (Park et al., 2001). The genomic sequence of CL-hepc has two introns and three exons, which is similar to that of other identified HEPCs in mammals and fish (Park et al., 2001; Pigeon et al., 2001; Li et al., 2011; Wang et al., 2009, 2012; Qu et al., 2013; Gong et al., 2014). Above all, CL-hepc is a member of the HEPC family.

The BLAST results of the deduced amino acid sequence of CL-hepc on the NCBI website shows that it belongs to the HEPC family, with $88.89 \%$ similarity with 83416593 L. crocea (ABC18307), 87.78\% similarity with $M$. dolomieu, $83.33 \%$ similarity with $P$. scotti, and relatively low similarity (47.73\%) with 158358729 L. crocea (ABY84845.1). In addition, the phylogenetic tree also shows that fish HEPCs are clustered in three branches and the two HEPCs of $L$. crocea are separated. Given such a close relationship with L. crocea (Tian et al., 2004), the presence of an additional type of HEPC in C. lucidus warrants further investigation.

Antimicrobial peptides form part of the main innate immune response components of fish defense against opportunistic pathogens (Ravichandran et al., 2010). The mRNA expression pattern of a gene is closely related to its function. In this study, we examined the expression of CLhepc in eight tissues, showing that expression was highest in the liver, which is seen as a common pattern in most HEPCs (Sang et al., 2006; Fu et al., 2007; Robertson et al., 2009; Oliveira Filho et 
al., 2010; Nam et al., 2011; Wang et al., 2012; Alvarez et al., 2013). In this sense, CL-hepc may prove to be an important component participating in innate immune activities. However, HEPCs are not expressed in the liver in channel catfish (Bao et al., 2005) or zebrafish (Shike et al., 2004), suggesting that they may be different isoforms. Based on the knowledge that the expression of PC-hepc (158358729 L. crocea) is high in the kidney and relatively low in the liver, we assume that CL-hepc and PC-hepc are not the same isoform. Although different, all these HEPCs play important roles in innate immune responses (Shike et al., 2004; Bao et al., 2005; Wang et al., 2009; Cai et al., 2012; Qu et al., 2013). In addition, peptides obtained from purified, recombinant, and artificial synthesis all exhibit effective activity against several pathogens (Park et al., 2001; Bao et al., 2005; Wang et al., 2009). Furthermore, it is encouraging that some drug-resistant strains, such as Aeromonas hydrophila, are still sensitive to the recombinant HEPC peptide (Gao et al., 2012), suggesting that a future study on HEPC is potentially promising.

\section{Conflicts of interest}

The authors declare no conflict of interest.

\section{ACKNOWLEDGMENTS}

Research supported by the Basic Research Fund for State-Level Nonprofit Research Institutes of ESCFRI, CAFS (\# Dong2012M06).

\section{REFERENCES}

Alvarez CA, Santana PA, Guzman F, Marshall S, et al. (2013). Detection of the hepcidin prepropeptide and mature peptide in liver of rainbow trout. Dev. Comp. Immunol. 41: 77-81.

Bao B, Peatman E, Li P, He C, et al. (2005). Catfish hepcidin gene is expressed in a wide range of tissues and exhibits tissuespecific upregulation after bacterial infection. Dev. Comp. Immunol. 29: 939-950.

Cai L, Cai JJ, Liu HP, Fan DQ, et al. (2012). Recombinant medaka (Oryzias melastigmus) pro-hepcidin: Multifunctional characterization. Comp. Biochem. Physiol. B Biochem. Mol. Biol. 161: 140-147.

Chen SL, Xu MY, Ji XS, Yu GC, et al. (2005). Cloning, characterization, and expression analysis of hepcidin gene from red sea bream (Chrysophrys major). Antimicrob. Agents Chemother. 49: 1608-1612.

Cheng J, Ma GQ, Miao ZQ, Shui BN, et al. (2012). Complete mitochondrial genome sequence of the spinyhead croaker Collichthys lucidus (Perciformes, Sciaenidae) with phylogenetic considerations. Mol. Biol. Rep. 39: 4249-4259.

Fry MM, Liggett JL and Baek SJ (2004). Molecular cloning and expression of canine hepcidin. Vet. Clin. Pathol. 33: 223-227.

Fu YM, Li SP, Wu YF and Chang YZ (2007). Identification and expression analysis of hepcidin-like cDNAs from pigeon (Columba livia). Mol. Cell Biochem. 305: 191-197.

Ganz T (2003). Hepcidin, a key regulator of iron metabolism and mediator of anemia of inflammation. Blood 102: 783-788.

Gao Y, Chen CF, Li DP, Tan JJ, et al. (2012). Cloning, expression and antimicrobial activity analysis of hepcidin from Chinese sturgeon (Acipenser sinensis). Acta Hydrobiol. Sin. 36: 798-803.

Gong LC, Wang H and Deng L (2014). Molecular characterization, phylogeny and expression of a hepcidin gene in the blotched snakehead Channa maculata. Dev. Comp. Immunol. 44: 1-11.

Hosaka M, Nagahama M, Kim WS, Watanabe T, et al. (1991). Arg-X-Lys/Arg-Arg motif as a signal for precursor cleavage catalyzed by furin within the constitutive secretory pathway. J. Biol. Chem. 266: 12127-12130.

Krause A, Neitz S, Mägert HJ, Schulz A, et al. (2000). LEAP-1, a novel highly disulfide-bonded human peptide, exhibits antimicrobial activity. FEBS Lett. 480: 147-150.

Li W, Sun WX, Tang F, Li CP, et al. (2011). Molecular characterization and expression analysis of a hepcidin gene from rice field eel (Monopterus albus). Afr. J. Biotechnol. 10: 7953-7961.

Magnadottir B (2006). Innate immunity of fish (overview). Fish Shellfish Immunol. 20: 137-151.

Nam YK, Cho YS, Lee SY, Kim BS, et al. (2011). Molecular characterization of hepcidin gene from mud loach (Misgurnus mizolepis; Cypriniformes). Fish Shellfish Immunol. 31: 1251-1258. 
Nemeth E, Valore EV, Territo M, Schiller G, et al. (2003). Hepcidin, a putative mediator of anemia of inflammation, is a type II acute-phase protein. Blood 101: 2461-2463.

Nemeth E, Tuttle MS, Powelson J, Vaughn MB, et al. (2004). Hepcidin regulates cellular iron efflux by binding to ferroportin and inducing its internalization. Science 306: 2090-2093.

Nicolas G, Chauvet C, Viatte L, Danan JL, et al. (2002). The gene encoding the iron regulatory peptide hepcidin is regulated by anemia, hypoxia, and inflammation. J. Clin. Invest. 110: 1037-1044.

Oliveira Filho JP, Badial PR, Cunha PHJ, Cruz TF, et al. (2010). Cloning, sequencing and expression analysis of the equine hepcidin gene by real-time PCR. Vet. Immunol. Immunopathol. 135: 34-42.

Park CH, Valore EV, Waring AJ and Ganz T (2001). Hepcidin, a urinary antimicrobial peptide synthesized in the liver. J. Biol. Chem. 276: 7806-7810.

Pigeon C, Ilyin G, Courselaud B, Leroyer P, et al. (2001). A new mouse liver-specific gene, encoding a protein homologous to human antimicrobial peptide hepcidin, is overexpressed during iron overload. J. Biol. Chem. 276: 7811-7819.

Qu H, Chen B, Peng H and Wang K (2013). Molecular cloning, recombinant expression, and antimicrobial activity of EChepcidin3, a new four-cysteine hepcidin isoform from Epinephelus coioides. Biosci. Biotechnol. Biochem. 77: 103-110.

Ravichandran S, Kumaravel K, Rameshkumar G and AjithKumar T (2010). Antimicrobial peptides from the marine fishes. Res. J. Immunol. 3: 146-156.

Robertson LS, Iwanowicz LR and Marranca JM (2009). Identification of centrarchid hepcidins and evidence that 17betaestradiol disrupts constitutive expression of hepcidin-1 and inducible expression of hepcidin- 2 in largemouth bass (Micropterus salmoides). Fish Shellfish Immunol. 26: 898-907.

Rodrigues PN, Vazquez-Dorado S, Neves JV and Wilson JM (2006). Dual function of fish hepcidin: response to experimental iron overload and bacterial infection in sea bass (Dicentrarchus labrax). Dev. Comp. Immunol. 30: 1156-1167.

Saitou N and Nei M (1987). The neighbor-joining method: a new method for reconstructing phylogenetic trees. Mol. Biol. Evol. 4: 406-425.

Sang Y, Ramanathan B, Minton JE, Ross CR, et al. (2006). Porcine liver-expressed antimicrobial peptides, hepcidin and LEAP2: cloning and induction by bacterial infection. Dev. Comp. Immunol. 30: 357-366.

Shike H, Lauth X, Westerman ME, Ostland VE, et al. (2002). Bass hepcidin is a novel antimicrobial peptide induced by bacterial challenge. Eur. J. Biochem. 269: 2232-2237.

Shike H, Shimizu C, Lauth X and Burns JC (2004). Organization and expression analysis of the zebrafish hepcidin gene, an antimicrobial peptide gene conserved among vertebrates. Dev. Comp. Immunol. 28: 747-754.

Sun M, Jiang K, Zhang F, Zhang D, et al. (2012). Effects of various salinities on $\mathrm{Na(+)-K(+)-ATPase,} \mathrm{Hsp70} \mathrm{and} \mathrm{Hsp90}$ expression profiles in juvenile mitten crabs, Eriocheir sinensis. Genet. Mol. Res. 11: 978-986.

Tian LX, Liang B, Zhang SY, Zhao S L, et al. (2004). Phylogenetic relationships of 7 Sciaenidae species based on cytochrome b gene sequences. J. Oceanogr. Taiwan Strait 23: 436-443.

Wang KJ, Cai JJ, Cai L, Qu HD, et al. (2009). Cloning and expression of a hepcidin gene from a marine fish ( $P$ seudosciaena crocea) and the antimicrobial activity of its synthetic peptide. Peptides 30: 638-646.

Wang KJ, Cai JJ and Cai L (2011). New hepcidin antibacterial peptide of large yellow croaker useful as a feed additive for preventing disease and treating sea fishes. Xiamen University, Xiamen.

Wang Y, Liu X, Ma L, Yu Y, et al. (2012). Identification and characterization of a hepcidin from half-smooth tongue sole Cynoglossus semilaevis. Fish Shellfish Immunol. 33: 213-219. 